\title{
Translation of a Circulating microRNA Signature of Melanoma to a Novel Solid-Tissue Biomarker to Improve Diagnostic Accuracy and Reproducibility.
}

Ryan Van Laar ( $\square$ rvanlaar@geneseq.com.au )

Geneseq Biosciences https://orcid.org/0000-0003-2828-4175

\section{Samuel King}

Australian Clinical Labs

Richard McCoy

Australian Clinical Labs

Mirette Saad

Australian Clinical Labs

Sian Fereday

Geneseq Biosciences

Ingrid Winship

Geneseq Biosciences

Catherine Uzzell

Australian Clinical Labs

Anthony Landgren

Australian Clinical Labs

Research article

Keywords: Diagnostic Accuracy, Reproducibility, Solid-Tissue Biomarker

Posted Date: December 4th, 2020

DOI: https://doi.org/10.21203/rs.3.rs-77357/v1

License: (1) (1) This work is licensed under a Creative Commons Attribution 4.0 International License.

Read Full License 


\section{Abstract \\ Background}

Successful treatment of cutaneous melanoma depends on early and accurate diagnosis of clinically suspicious melanocytic skin lesions. Currently, histopathology examination of excised skin lesions is considered the 'gold standard' for diagnosis of melanoma. Multiple studies have shown the low accuracy and reproducibility of this method, underscoring the urgent need for new diagnostic tools, including disease-specific biomarkers. Previously, a 38-microRNA signature of melanoma ('Mel38') was previously identified in plasma and validated as novel circulating biomarker. In this study, Mel38 expression in solid biopsy tissue is examined to determine its ability to contribute to accurate and reproducible melanoma diagnoses.

\section{Methods}

Nanostring digital gene expression profiling was used to apply the Mel38 signature in a cohort of 308 formalin fixed paraffin embedded skin biopsies ('Mel38'). Genomic data were interrogated using hierarchical clustering, univariate and multivariate statistical approaches. Mel38 classification scores (range 0 to 10) were compared to consensus histopathology results, including MPATH-DX class, AJCC disease stage, histological subtype as well as technical assay factors.

\section{Results}

The Mel38 score can identify high-risk melanomas (MPATH-Dx Class IV) from less-malignant forms of the disease with an area-under-the curve of $0.96(P<0.001)$. The genomic score ranges from 0 to 10 and is positively correlated with the melanoma progression, from benign naevi to metastatic disease (intraclass correlation coefficient: 0.85 ). Using a score threshold of $>2.3$ identifies higher-risk melanomas, associated with poorer outcomes and more intensive suggested clinical actions. Multivariate analysis showed the score to be a significant predictor of malignancy, independent of technical and clinical covariates. Analysis of the Mel38 signature in spitz naevi reveal an intra-subtype profile, in common to both benign and malignant conditions.

\section{Conclusion}

Melanoma-specific circulating microRNAs maintain their association with malignancy when measured in the hypothesized tissue of origin. The Mel38 signature is an accurate and reproducible metric of melanoma status, based on changes in microRNA expression that occur as the disease develops and spreads. Inclusion of the Mel38 score into routine practice would give physicians a genomic assessment 
of a patient's disease status. Combining molecular biomarker data with conventional histopathology data may improve diagnostic accuracy, reproducibility, and patient outcomes.

\section{Background}

Over the past two decades, the annual incidence of melanoma has increased by $4-6 \%$ in fair-skinned populations of Northern Europe, North America, New Zealand and Australia[1]. Australia leads the world in melanoma incidence, with two out of three people diagnosed with some form of skin cancer during their lifetime. Unfortunately, public awareness campaigns nor advances in diagnosis and therapeutics have translated to prolonged improvements in the age-standardised mortality rates for this cancer type in many countries[2].

Currently, melanocytic lesions suspected of being malignant are diagnosed almost exclusively by visual methods, including dermoscopy, followed by biopsy and histopathologic examination. Advances in detection and diagnosis have increased the incidence of melanoma diagnoses worldwide but have not reduced the mortality rate. There is consensus among experts that standardisation of diagnostic terminology and new molecular tests are urgently needed to improve patient outcomes.

The MPATH-Dx structure for describing melanocytic lesions is one method that is being adopted by pathologists as method to improve diagnostic performance and consistency between laboratories $[3,4]$. Despite this and other improvements in related areas such as dermoscopy, multiple studies into the accuracy and reproducibility of melanoma diagnosis by histopathology alone is "not accurate nor reproducible" [5]. Concerningly, results from the Elmore et al study showed that $637 / 2283$ (28\%) of MPATH-Dx Class $V$ melanomas (those requiring the widest excision margins and consideration of lymph node screening and/or adjuvant therapy) were under-diagnosed by pathologists in the study.

Reproducibility of Class V diagnoses was also shown to be challenging, with 179/1031 (17\%) of these melanomas receiving a lower classification when re-analysed by the same pathologist.

Genomic profiling of microRNAs is a molecular technique that can be performed on many tissue types, including blood and skin biopsy material. MicroRNAs are transcription-regulating nucleic acids which have tissue- and disease-specific patterns of expression. Their role in melanomagenesis and progression is well documented, including a crucial role in formation of the dermal tumour niche, as well as recruitment and activation of cancer associated fibroblasts via exosomal secretion, necessary for invasion and metastatic spread[6].

Previously, a novel signature of 38 circulating microRNAs ('Mel38') was identified by genomic profiling of plasma from individuals with stage I-IV melanoma versus healthy controls. The biological functions of the Mel38 microRNAs include regulators of angiogenesis \& inflammation $(n=2)$, invasion \& metastasis $(n=14)$, immune response / treatment resistance $(n=11)$ and tumour suppression or oncogene activation $(n=8)[7,8]$. To express the information represented by the Mel38 signature in an interpretable and patientpersonalised form, a gene weighting algorithm was used to compute a classification score (ie. Mel38 score) that ranges from 0 to 10, positively associated with increasing malignancy. In multiple validation 
cohorts comprised of varied specimen types, the Mel38 score exhibited a robust and statistically significant association with melanoma status, treatment and prognosis[9].

In this study we perform microRNA analysis of 308 clinically annotated, formalin fixed paraffin embedded melanocytic skin lesions, representing the specimen type and range of histological subtypes commonly analysed in skin pathology practices. The clinical and technical accuracy of the Mel38 signature is evaluated and contrasted with equivalent metrics derived for current diagnostic techniques.

\section{Patients And Methods}

\section{Sample population}

This retrospective study was performed using melanocytic lesions that were submitted for diagnostic assessment (Australian Clinical Laboratories) as part of normal health care over the prior five years. Specimens were selected to represent the progression from benign naevi through to advanced invasive adult melanoma (18 years and older), including multiple common histological subtypes and disease stages (AJCC $8^{\text {th }}$ Ed[10]). An additional 14 samples of spitz naevi from patients younger than 18 at the time of diagnosis were also included.

Each specimen was reviewed by two or more experienced dermatopathologists, with the majority diagnosis recorded as the result. The study was approved by Australian Clinical Labs internal medical advisory board and satisfies criteria for use of human tissue by diagnostic pathology companies as outlined by the Australian Government's National Health and Medical Research Council [11]. A study sample size target of $>300$ specimens was set, was based on the method described by Hajian-Tilak, assuming an AUC of 0.78 or higher and the detection of a small-moderate effect size with $80 \%$ power and 95\% confidence[12].

\section{RNA extraction and genomic analysis}

Each FFPE specimen required two or more unstained slides containing a total of $20 \mu \mathrm{m}$ of tissue and $>=10 \%$ naevi or $20 \%$ melanoma cell content. A representative area of each specimen was circled on the $\mathrm{H} \& \mathrm{E}$ slide by the reviewing pathologist and macro-dissected from the unstained slides. Total RNA was extracted from deparaffinated tissue using the miRNeasy FFPE kit (QIAGEN Cat No./ID: 217504) and quantified using a Nanodrop 2000 (ThermoFisher, USA). MicroRNA profiling was performed using the Nanostring nCounter Human v3 miRNA Panel with the nCounter SPRINT or nCounter MAX platform (Nanostring Inc, Seattle USA), according to the manufacturer's guidelines.

\section{Data processing and statistical analysis}


Log2 scaled raw data were adjusted for technical variation by positive control scaling and 'top 100' normlisation using the NanoStringNorm R library [13]. Normalised data were then examined for quality by comparing with predetermined internal standards, before a classification score ('Mel38 score') was calculated for each specimen using the gene weighting algorithm as previously described [8]. A representative subset of nevi and invasive melanoma samples $(n=24)$ was used to update the gene weights used to compute the classification score from FFPE data.

Data normalisation, quality control and Mel38-FFPE score calculations were completed using R. Statistical analysis and visualisation performed using Microsoft Excel and MedCalc [14, 15]. All P-values calculated are two-sided and when $<0.05$ were considered to be statistically significant. Receiver operator curve (ROC) analysis was used to assess the sensitivity and specificity of the Mel38-FFPE score and determine the optimal score threshold to classify specimens as either high or low risk of disease progression and a potentially poorer outcome[16]. Binary logistic regression was used visualise the score as a continuous predictor of malignancy.

\section{Results}

\section{Genomic profiling of melanocytic lesions representing the spectrum of benign melanocytic lesion to metastatic melanoma.}

The Mel38 signature was examined using total RNA from 308 FFPE tissue specimens with a melanocytic cellular content of $10 \%$ or greater. Patient and specimen details are summarised in Table 1. To visualise the relationship between the 38 microRNAs and specimen details, two-dimensional hierarchical clustering of the complete cohort was performed (Figure 1). This resulted in an ordering of samples corresponding to an increasing degree of malignancy (left to right). The gradual transition in levels of relative up and down microRNA expression reflects the continuum of naevi to melanoma progression which is in clinical practice.

Notably, two microRNAs (hsa-mir-205 and hsa-mir-497) appear to have similarly low expression in the vastly different disease stats of benign naevi and invasive/metastatic melanoma, but high expression in early stage invasive disease. These microRNAs may therefore have a specific role in aiding a melanoma cell to develop invasive characteristics and warrant further investigation.

\section{The Mel38 score is positively correlated with increasing melanoma stage and is statistically significant independent to other variables.}


Mel38 scores for each specimen were calculated using support vector machine derived gene weights, as previously described. The scores range from 0 to 10 and are positivity correlated with the degree of malignancy, as shown by box plots of the scores vs AJCC stage (all samples) and vs MPath-Dx classes (primary lesions only), in figure Figure $3 \mathrm{~A}$ and $3 \mathrm{~B}$ respectfully. The intraclass correlation of the score vs specimen status (naevi to metastatic melanoma) was 0.85 .

To verify that the Mel38 score is a continuous predictor of malignancy, independent to other clinicopathological variables, general linear models (GLM) were computed using was using patient age, gender, histological subtype, and AJCC Stage or MPATH-Dx class[3]. In both models, the genomic score was significantly different between AJCC stages and MPATH-Dx classes, independent to the other variables in the model $(P<0.001)$.

A separate multivariate analysis of Mel38 scores from invasive melanoma specimens only $(n=128)$ was performed, including, Breslow depth, tumour cell content, patient age, gender and biopsy site. In this subset, the Mel38 score remained statistically significantly between disease stages $(P=0.012)$, independent of the other variables.

\section{The Mel38 score is an accurate binary classifier of clinically higher- vs. lower-risk melanocytic lesions.}

Whilst the Mel38 score demonstrates a statistically significant continuous association with malignancy, inspection of the MPATH-Dx vs Mel38 box plot (Figure $X$ ) shows that the largest difference in genomic scores is between MPATH-Dx Class IV and V specimens. Notable differences in suggested clinical actions and patient outcome (i.e. 5- and 10-year disease specific survival rates) are also observed between these two classes, as summarised in Table 2. Consequently, we sought to determine the accuracy of Mel38 as a binary classification tool of higher-risk vs lower-risk primary melanoma/naevi, i.e. Class V vs. i.e. Classes I-IV.

Receiver operator curve (ROC) analysis on Mel38 scores from 74 MPATH-Dx Class V and 181 Class I-IV specimens was performed and resulted in area under the curve (AUC) was 0.96 (95\% $\mathrm{Cl} 0.92$ to 0.98 , $\mathrm{P}<0.001$ ). Inspection of the ROC data showed that a Mel38 score threshold of $\geq 2.3$ (= higher-risk) corresponded to a true positive rate of $95 \%$ (ie. Sensitivity; $95 \mathrm{Cl}: 87 \%$ to $99 \%$ ) and true negative rate of $83 \%$ (i.e. specificity; Cl: $77 \%$ to $89 \%$ ). When evaluated in the context of MPATH-Dx Class V melanomas, using a Mel38 score threshold of $>2.3$ would result in a underdiagnosis rate of $8.8 \%$ (10/113), which is $2 / 3^{\text {rd }}$ lower than underdiagnosis rate of $27 \%$ for conventional pathology alone[5].

\section{Mel38 profiling of Spitz naevi shows similarity to both benign and invasive melanoma.}


Spitz naevi are an uncommon type of melanocytic naevus, histologically similar to melanoma and regarded as a challenging subcategory of melanocytic skin lesions to diagnose. Mel38-FFPE analysis was performed on twenty specimens of Spitz naevi submitted for routine histopathologic analysis from individuals ranging from two to thirty years old.

As shown in Figure 1, the expression profile of the spitz naevi exhibits similarity to both benign and malignant lesions. Inspection of individual microRNAs in this figure reveals several that appear to have spitz-specific patterns of expression, i.e. hsa-mir-424-5p, hsa-mir-301a-3p and hsa-mir-1537-3p.

One way ANOVA of the Mel38 scores in spitz naevi vs other naevi subtypes revealed statistically significance $(P<0.001)$, with mean scores of 2.7 (95\% Cl: 2.5 to 3.0$)$ vs 1.5 ( $95 \% \mathrm{Cl}$ : 1.4 to 1.7$)$, respectively, and significant pairwise differences to all naevi subtypes present. When analysed using a multivariate GLM, incorporating patient age, gender, and naevi subtype, the Mel38 scores of the spitz naevi were only significantly higher than the compound naevi subtype $(P=0.015)$. This suggests that the younger age of the spitz naevi patients compared to the rest of the cohort may be an influence on the Mel38 expression profile.

\section{The Mel38 score exhibits low technical variability between replicates and in longitudinal control sample analysis.}

RNA from 30 naevi and 30 melanoma samples was pooled and analysed over a period of 7 (nonsequential) weeks. The risk scores of each pool are shown longitudinally in Figure 5A. The mean score for the naevi control pool is 2.1 (standard deviation: 0.25 ) and 6.5 (standard deviation: 0.15 ) for the invasive melanoma pool.

To assess the reproducibility of the genomic score throughout its dynamic range (i.e. 0 to 10), RNA from 89 samples was re-analysed and compared to the original Mel38 score. As shown in Figure 5B, there is high consistency between replicate scores, with an intraclass correlation coefficient of $0.96,(95 \% \mathrm{Cl} 0.94$ to 0.98 ) and no deviation from linearity (Cusum test, $P=0.62$ ).

These results show that the Mel38 signature exhibits a high degree of longitudinal stability and technical reproducibility.

\section{Discussion}

Melanoma is a heterogeneous, progressive disease. It originates from melanocytic skin lesions and progresses through a series of well-documented stages of malignancy. In this study we describe the performance of a novel genomic biomarker for melanoma in a different specimen type (FFPE biopsy tissue) from that in which it was discovered (plasma). By comparing the 38-microRNA signature to relevant clinicopathological and specimen variables, its potential to contribute accurate and reproducible information to a patient's diagnostic picture is demonstrated. 
The cohort presented in this study was designed to reflect the clinical continuum of melanoma and enabled the resulting data to be analysed both as a spectrum disease, from benign naevi to metastatic melanoma, as well a binary condition, by classifying specimens into MPATH-Dx classes. In the former context, the Mel38 score exhibits a strong positive correlation with the progressive stages of melanoma, as represented by the ICC coefficient (0.85). When analysed as binary condition, with groups formed based on preestablished diagnostic classes with substantially different recommended follow-up actions and associated patient outcomes, a Mel38 threshold of $>2.3$ was able to identify higher risk disease with high sensitivity and specificity.

A selection of spitz naevi specimens were included in this study due to their histological similarity with melanoma. Despite the fact they are usually diagnosed in younger patients, misdiagnosis of melanoma as a spitz nevus is an ongoing challenge[17]. Hierarchical clustering showed the expression profile of this subtype to have similaries to both benign and malignant disease, which was also reflected by their Mel38 scores. To date very few genomic studies of spitz naevi have been performed; only 56 out of 47,333 $(0.1 \%)$ melanocytic lesion profiles in the NCBI Gene Expression Omnibus database are of spitzoid type[18]. This observation, albeit based on a limited sample size, may indicate there are a subset of spitz naevi which may require additional treatment or monitoring.

Technical reproducibility of MEL38 signature was demonstrated by the low level of variation observed for both a melanoma and naevi control RNA pool, tested repeatedly over several months. Low variation and strong linearity was observed between a series of samples covering the full dynamic range of the score which were analysed in duplicate. These technical data are in line with those of other well validated genomic signatures used for individual patient management. Finally, the reproducibility of the Mel38 demonstrated in these analyses well exceeds that of conventional pathology based diagnoses, where almost one third of specimens may receive a different MPATH-Dx class when re-reviewed by the same pathologist[5].

Limitations of this study is the small numbers of less-common melanoma and naevi subtypes, such as amelanotic melanoma. Future work will focus on specific subtypes of melanoma and non-cancerous skin lesions which present challenges to accurately diagnose using conventional methods. A further limitation is not directly associating the genomic score to patient outcome, due to the lack access to this information.

It is important to highlight the proposed use of Mel38-FFPE is as a complementary molecular biomarker to conventional histopathology, and not intended to replace current practice. Genomic testing, microscopic and immunohistochemical assessments of cellular/tissue structure all add unique, but only partially overlapping, data points to a patient's overall diagnostic picture. The combination of diagnostic modalities is becoming standard of care for many cancer types including breast (eg. Oncotype DX, MammaPrint, EndoPredict) and prostate (Oncotype DX, Prolaris, ProstaVysion), both of which have seen promising reductions in mortality over recent years. 
Clinical adaptation of Mel38-FFPE has the potential to add novel, robust and personalised genomic information to the diagnostic picture of patients with clinically suspicious melanocytic lesions.

\section{Conclusion}

Successful treatment of melanoma begins with accurate evaluation of melanocytic skin lesions. The consequences of over-or under-diagnosis could be avoided by using complementary diagnostic techniques. The Mel38 genomic signature, now validated in FFPE tissue, demonstrates a high degree of clinical accuracy and technical reproducibility, making it suitable for use as an adjunct diagnostic biomarker in the clinical setting.

\section{Abbreviations}

Mel38: 38 microRNA signature.

GLM: General linear model.

FFPE: Formalin fixed paraffin embedded.

\section{Declarations}

\section{Source of funding:}

Institutional funding.

\section{Disclosures:}

\section{Author abbreviations}

Ryan Van Laar: RVL, Samuel King: SK, Amicel Baynosa: AB, Richard McCoy: RM, Mirette Saad: MS, Sian Fereday: SF, Ingrid Winship: IW, Catherine Uzzell: CU, Anthony Landgren: AL.

\section{Ethics approval and consent to participate.}

The study was approved by Australian Clinical Labs internal medical advisory board and satisfies criteria for use of human tissue by diagnostic pathology companies as outlined by the Australian Government's National Health and Medical Research Council.

\section{Consent for publication}

N/A

\section{Availability of data and materials}

Data available upon request 


\section{Declaration of interest}

RVL: Employee and shareholder in Geneseq Biosciences.

IW: Board Member and Shareholder in Geneseq Biosciences

SF: Consultant to Geneseq Biosciences

SK, RM, MS, CU, AL: Employee of Australian Clinical Labs

\section{Funding}

NA

\section{Authors' contributions}

Study design and management: RVL, MS, IW, CU, AL

Laboratory work: SK, AM, RM

Pathology review: $\mathrm{CU}, \mathrm{AL}$

Data analysis: RVL, SF

Manuscript drafting and review: All.

\section{Acknowledgements}

The authors acknowledge the assistance of Amicel Baynosa and Keith Byron in the specimen processing and study initiation.

\section{References}

1. Matthews, N.H., et al., Epidemiology of Melanoma, in Cutaneous Melanoma: Etiology and Therapy, W.H. Ward and J.M. Farma, Editors. 2017: Brisbane (AU).

2. Australian Institute of Health and, W. and R. Australasian Association of Cancer, Cancer survival in Australia, 2001: [relative survival data for selected cancers for the period 1982 to 1997]. 2001, Canberra: Australian Institute of Health and Welfare. 3.

3. Piepkorn, M.W., et al., The MPATH-Dx reporting schema for melanocytic proliferations and melanoma. J Am Acad Dermatol, 2014. 70(1): p. 131-41.

4. Radick, A.C., et al., Terminology for Melanocytic Skin Lesions and the MPATH-Dx Classification Schema: A Survey of Dermatopathologists. J Cutan Pathol, 2020.

5. Elmore, J.G., et al., Pathologists' diagnosis of invasive melanoma and melanocytic proliferations: observer accuracy and reproducibility study. BMJ, 2017. 357. 
6. Dror, S., et al., Melanoma miRNA trafficking controls tumour primary niche formation. Nat Cell Biol, 2016. 18(9): p. 1006-17.

7. van Laar, R.K., M.T. Linpcoln, and B.J. van Laar, A plasma microRNA biomarker of melanoma as a personalised assessment of treatment response. Melanoma Res, 2019. 29(1): p. 19-22.

8. Van Laar, R., M. Lincoln, and B. Van Laar, Development and validation of a plasma-based melanoma biomarker suitable for clinical use. Br J Cancer, 2018. 118(6): p. 857-866.

9. Van Laar, R., M. Lincoln, and S. Fereday, Characterisation and validation of Mel38; A multi-tissue microRNA signature of cutaneous melanoma. PLoS One, 2019. 14(2): p. e0211504.

10. Gershenwald, J.E., et al., Melanoma staging: Evidence-based changes in the American Joint Committee on Cancer eighth edition cancer staging manual. CA Cancer J Clin, 2017. 67(6): p. 472492.

11. Sainsbury, P., Development and oversight of ethical health promotion quality assurance and evaluation activities involving human participants. Health Promot J Austr, 2015. 26(3): p. 176-181.

12. Hajian-Tilaki, K., Sample size estimation in diagnostic test studies of biomedical informatics. J Biomed Inform, 2014. 48: p. 193-204.

13. Waggott, D., et al., NanoStringNorm: an extensible R package for the pre-processing of NanoString mRNA and miRNA data. Bioinformatics, 2012. 28(11): p. 1546-8.

14. Schoonjans, F., et al., MedCalc: a new computer program for medical statistics. Comput Methods Programs Biomed, 1995. 48(3): p. 257-62.

15. $R$ : A language and environment for statistical computing, ed. R.D.C. Team. 2010: R Foundation for Statistical Computing.

16. YOUDEN, W.J., Index for rating diagnostic tests. Cancer, 1950. 3(1): p. 32-5.

17. Gerami, P., et al., Histomorphologic assessment and interobserver diagnostic reproducibility of atypical spitzoid melanocytic neoplasms with long-term follow-up. Am J Surg Pathol, 2014. 38(7): p. 934-40.

18. Edgar, R., M. Domrachev, and A.E. Lash, Gene Expression Omnibus: NCBI gene expression and hybridization array data repository. Nucleic Acids Res, 2002. 30(1): p. 207-10.

19. Westgard, J.O., et al., A multi-rule Shewhart chart for quality control in clinical chemistry. Clin Chem, 1981. 27(3): p. 493-501.

\section{Tables}

Table 1: Patient and specimen demographics 


\begin{tabular}{|c|c|c|}
\hline Descriptor & Number & Percent \\
\hline \multicolumn{3}{|l|}{ Age: } \\
\hline Mean & 60 & \\
\hline$<30$ & 19 & $7 \%$ \\
\hline $31-40$ & 37 & $13 \%$ \\
\hline $41-50$ & 34 & $12 \%$ \\
\hline $51-60$ & 41 & $14 \%$ \\
\hline$>60$ & 157 & $54 \%$ \\
\hline \multicolumn{3}{|l|}{ Gender } \\
\hline Male: & 154 & $53 \%$ \\
\hline Female: & 134 & $47 \%$ \\
\hline \multicolumn{3}{|l|}{ Naevi } \\
\hline Superficial spreading & 1 & $1 \%$ \\
\hline Intradermal & 16 & $17 \%$ \\
\hline Compound & 37 & $40 \%$ \\
\hline Junctional & 16 & $17 \%$ \\
\hline Spitz & 20 & $22 \%$ \\
\hline Other & 3 & $3 \%$ \\
\hline \multicolumn{3}{|l|}{ Melanoma in-situ } \\
\hline Lentigo maligna & 33 & $69 \%$ \\
\hline Superficial spreading & 6 & $12 \%$ \\
\hline Other & 9 & $19 \%$ \\
\hline \multicolumn{3}{|l|}{ Invasive melanoma } \\
\hline Superficial spreading & 84 & $50 \%$ \\
\hline Nodular & 29 & $17 \%$ \\
\hline Lentigo maligna & 8 & $5 \%$ \\
\hline Other & 46 & $28 \%$ \\
\hline \multicolumn{3}{|l|}{ AJCC Clinical Stage: } \\
\hline IA & 58 & $35 \%$ \\
\hline
\end{tabular}




\begin{tabular}{|lll|} 
II IA & 19 & $11 \%$ \\
IIB & 17 & $10 \%$ \\
IIC & 21 & $13 \%$ \\
III/IV & 13 & $8 \%$ \\
MPATH-Dx Class & 39 & $23 \%$ \\
I & 33 & \\
II & $11 \%$ \\
III & 44 & $15 \%$ \\
IV & 50 & $17 \%$ \\
V & 54 & $18 \%$ \\
\hline
\end{tabular}

Table 2: MPATH-Dx classes, mean Mel38 scores and associated clinicopathological variables, including suggested clinical actions.*Melanoma specific survival figures based on AJCC melanoma staging and outcome data corresponding to the T-stage associated with each MPath-Dx class. "Suggested clinical actions shown for each class reproduced from Piepkorn et al [3] 


\begin{tabular}{|c|c|c|c|c|c|}
\hline $\begin{array}{l}\text { MPATH-Dx } \\
\text { Class: }\end{array}$ & I & II & III & IV & V \\
\hline $\begin{array}{l}\text { Mel38 score } \\
\text { (mean) }\end{array}$ & 1.5 & 1.7 & 1.7 & 2.1 & 5.2 \\
\hline T stage & $\mathrm{N} / \mathrm{A}$ & $\mathrm{N} / \mathrm{A}$ & 0 & $\mathrm{~T} 1 \mathrm{a}$ & T1b or more \\
\hline $\begin{array}{l}\text { Perceived } \\
\text { risk for } \\
\text { progression }\end{array}$ & $\begin{array}{l}\text { Very low } \\
\text { risk }\end{array}$ & Low risk & Higher risk & $\begin{array}{l}\text { Substantial } \\
\text { risk for } \\
\text { local/regional } \\
\text { progression }\end{array}$ & $\begin{array}{l}\text { Greatest risk for } \\
\text { regional and/or } \\
\text { distant } \\
\text { metastases }\end{array}$ \\
\hline $\begin{array}{l}5 \text { /10-year } \\
\text { melanoma- } \\
\text { specific } \\
\text { survival* }\end{array}$ & $\begin{array}{l}100 \% / \\
100 \%\end{array}$ & $\begin{array}{l}100 \% / \\
100 \%\end{array}$ & $100 \% / 100 \%$ & $98 \% / 96 \%$ & $\leq 93 \% / \leq 89 \%$ \\
\hline $\begin{array}{l}\text { Suggested } \\
\text { clinical } \\
\text { action\#: }\end{array}$ & $\begin{array}{l}\text { No further } \\
\text { treatment. }\end{array}$ & $\begin{array}{l}\text { Narrow but } \\
\text { complete } \\
\text { excision } \\
(<5 \mathrm{~mm})\end{array}$ & $\begin{array}{l}\text { Complete excision } \\
\text { with at least } 5 \\
\text { mm but }<10 \mathrm{~mm} \\
\text { margins. }\end{array}$ & $\begin{array}{l}\text { Wide local } \\
\text { excision with } \\
\geq 10 \mathrm{~mm} \\
\text { margins. }\end{array}$ & $\begin{array}{l}\text { Wide local } \\
\text { excision with } \\
\geq 10 \text { mm margins. } \\
\text { Consideration of } \\
\text { sentinel lymph } \\
\text { node biopsy, } \\
\text { adjuvant therapy. }\end{array}$ \\
\hline $\begin{array}{l}\text { Clinical } \\
\text { action likely } \\
\text { to require } \\
\text { referral: }\end{array}$ & No & & & & Yes \\
\hline
\end{tabular}

\section{Figures}




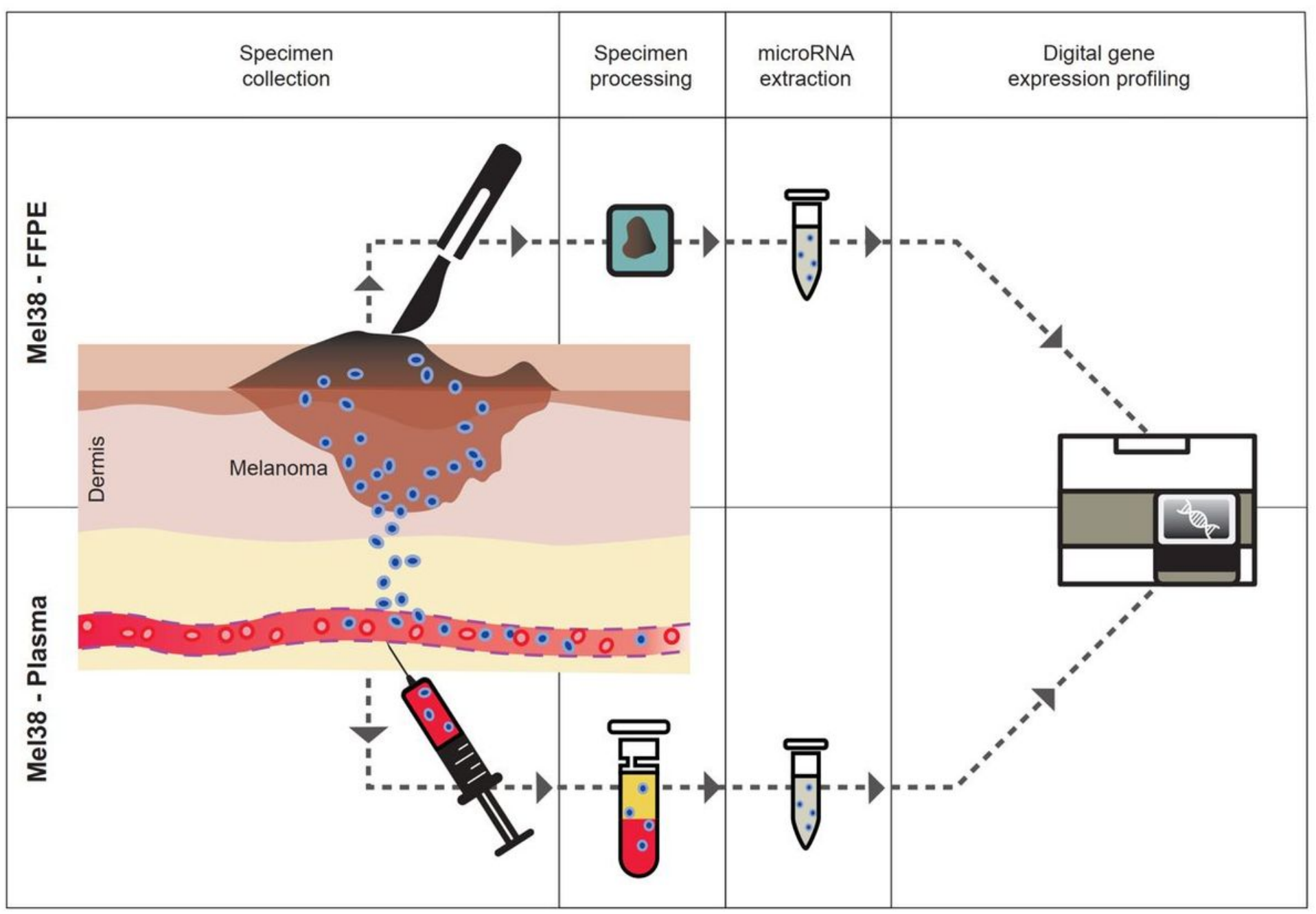

\section{Figure 1}

Schematic diagram of the development of Mel38, a microRNA signature of melanoma originally identified in plasma (lower section), and its adaptation to FFPE melanocytic skin lesions (upper section) using digital gene expression profiling methods and classification algorithms previously described. 


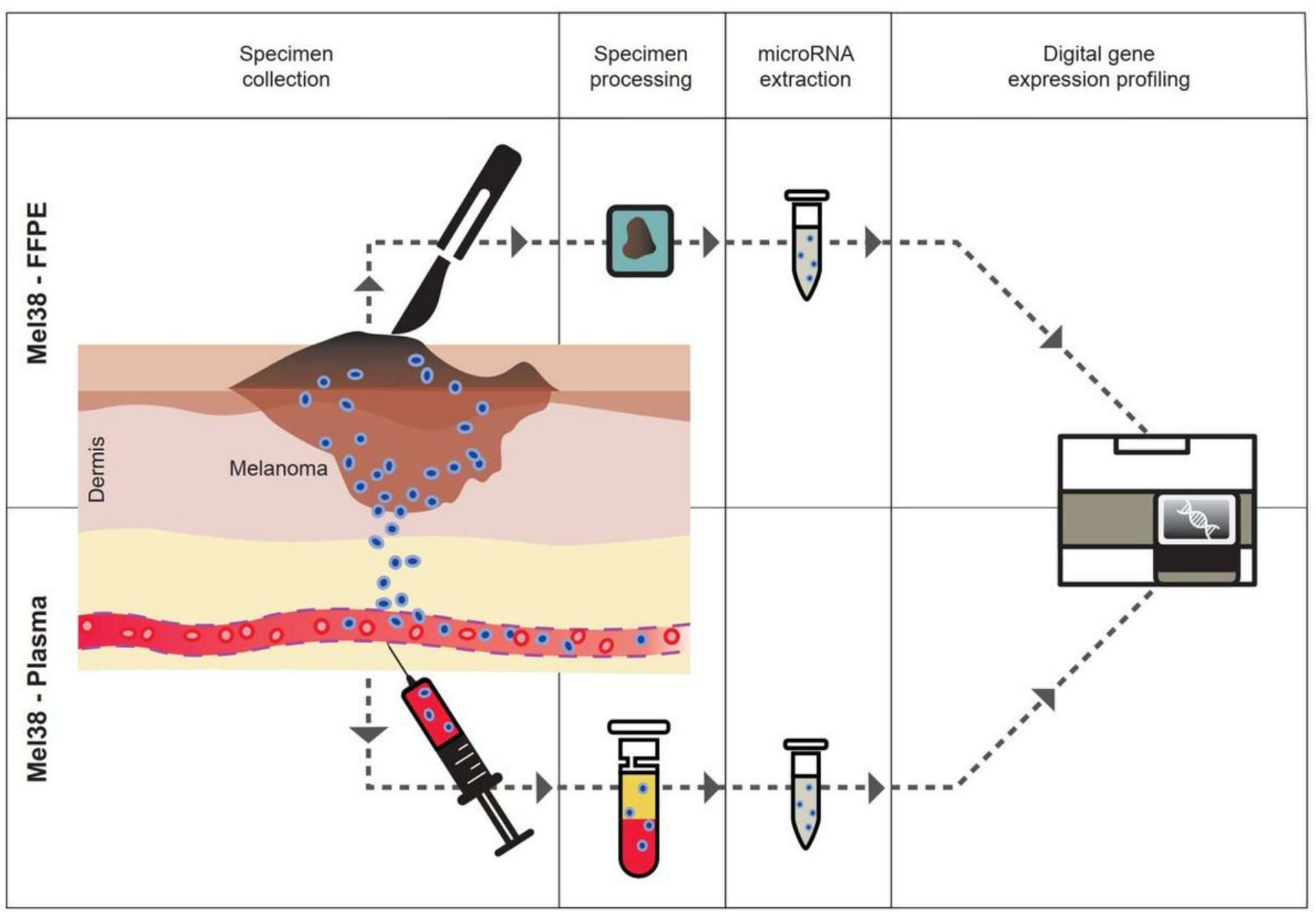

\section{Figure 1}

Schematic diagram of the development of Mel38, a microRNA signature of melanoma originally identified in plasma (lower section), and its adaptation to FFPE melanocytic skin lesions (upper section) using digital gene expression profiling methods and classification algorithms previously described. 


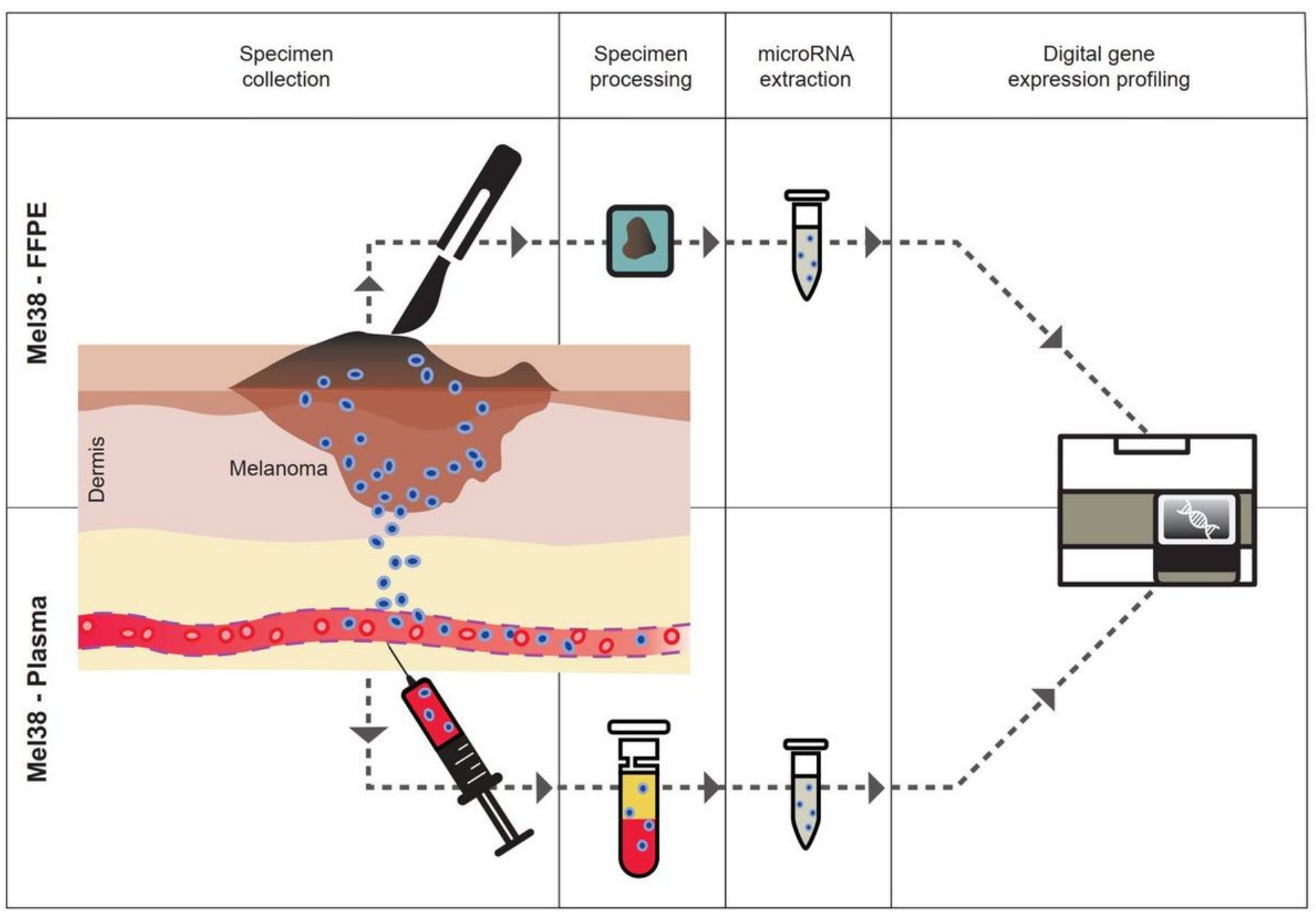

\section{Figure 1}

Schematic diagram of the development of Mel38, a microRNA signature of melanoma originally identified in plasma (lower section), and its adaptation to FFPE melanocytic skin lesions (upper section) using digital gene expression profiling methods and classification algorithms previously described. 


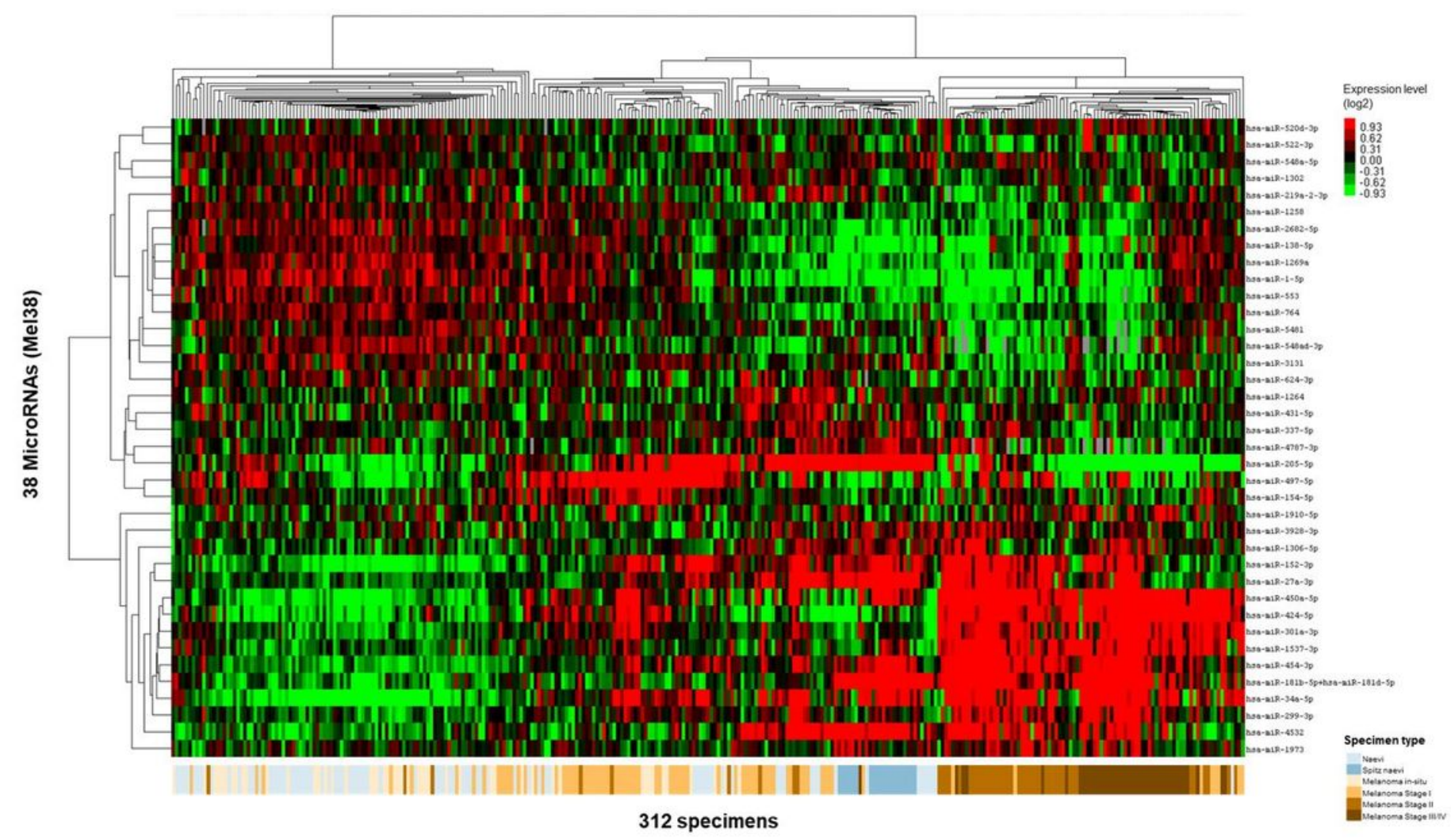

Figure 2

Supervised hierarchical clustering of the Mel38 signature data from 308 FFPE specimens of benign naevi, melanoma in-situ and invasive melanoma. The clustering pattern revealed shows blocks of up and down regulation, reflecting the raw data used by the Mel38-FFPE classifier to compute each patient's individual Mel38-FFPE score. 


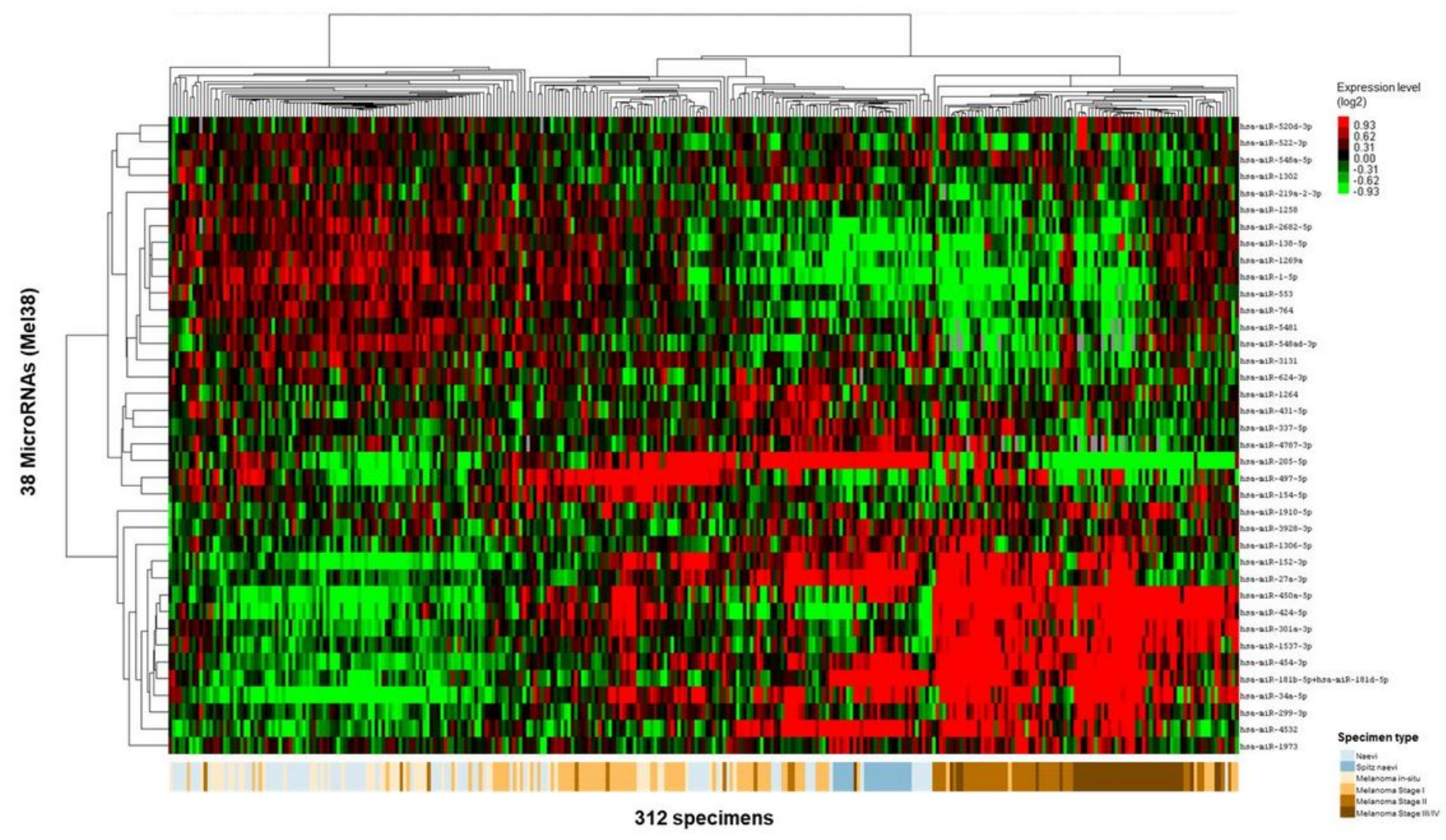

Figure 2

Supervised hierarchical clustering of the Mel38 signature data from 308 FFPE specimens of benign naevi, melanoma in-situ and invasive melanoma. The clustering pattern revealed shows blocks of up and down regulation, reflecting the raw data used by the Mel38-FFPE classifier to compute each patient's individual Mel38-FFPE score. 


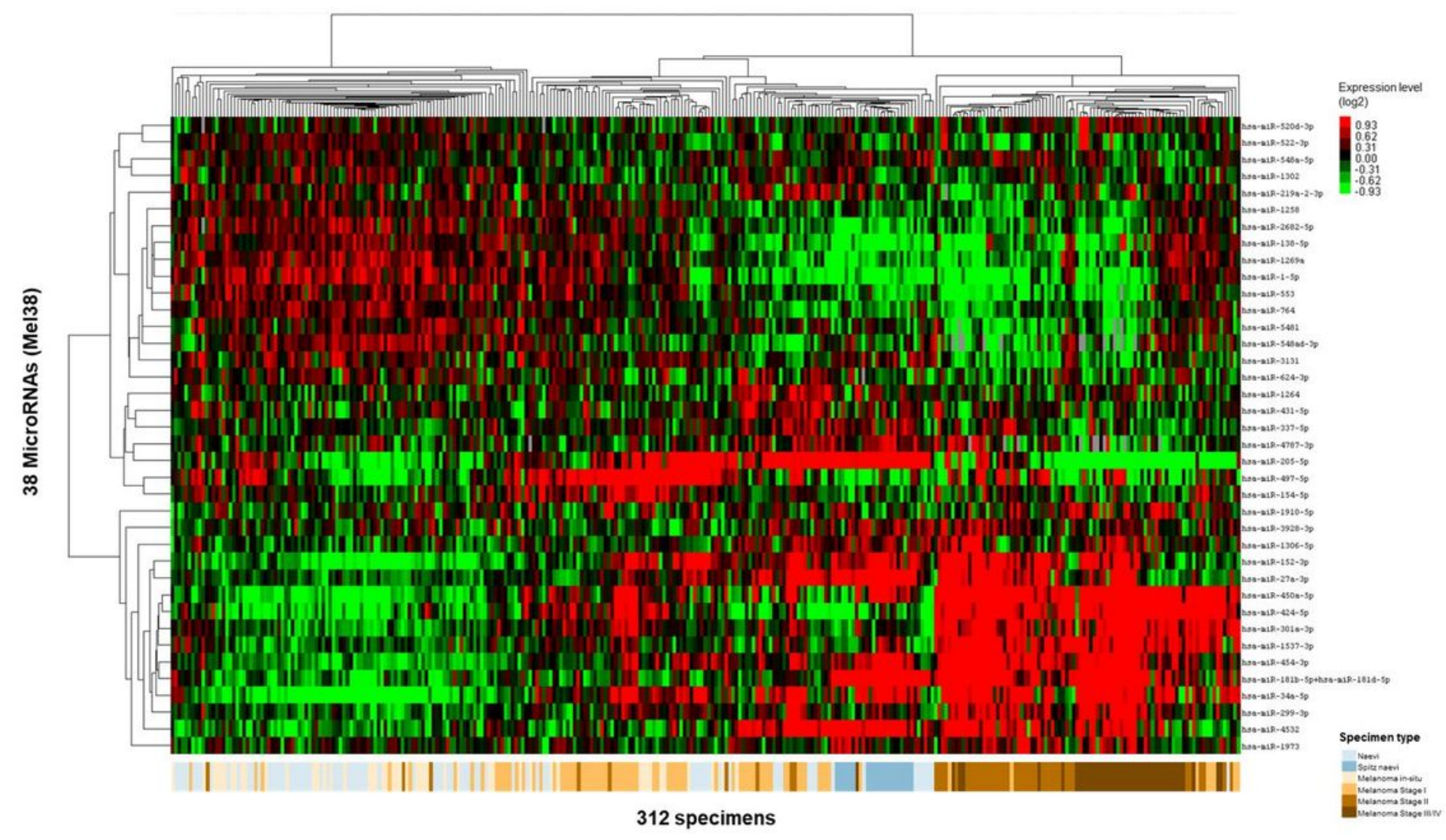

Figure 2

Supervised hierarchical clustering of the Mel38 signature data from 308 FFPE specimens of benign naevi, melanoma in-situ and invasive melanoma. The clustering pattern revealed shows blocks of up and down regulation, reflecting the raw data used by the Mel38-FFPE classifier to compute each patient's individual Mel38-FFPE score.

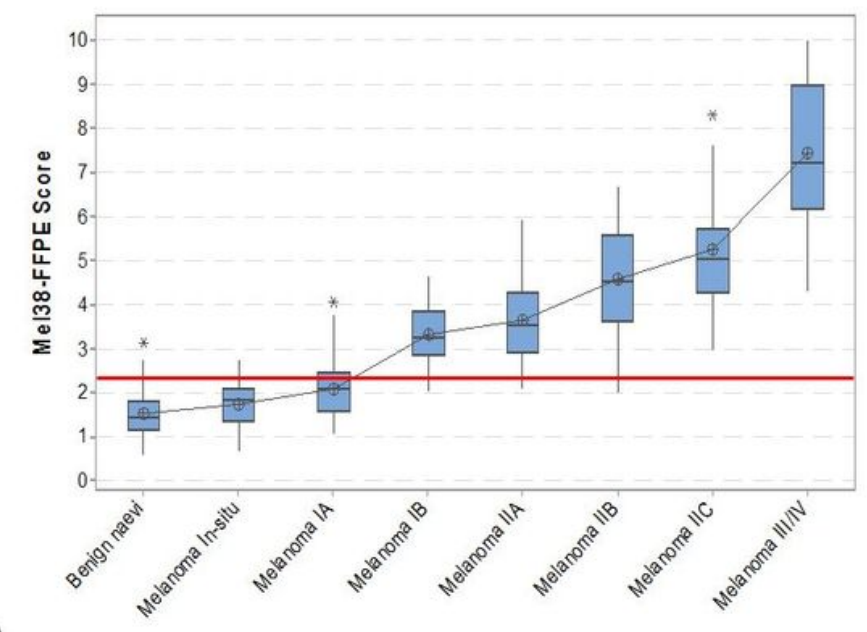

$B$.

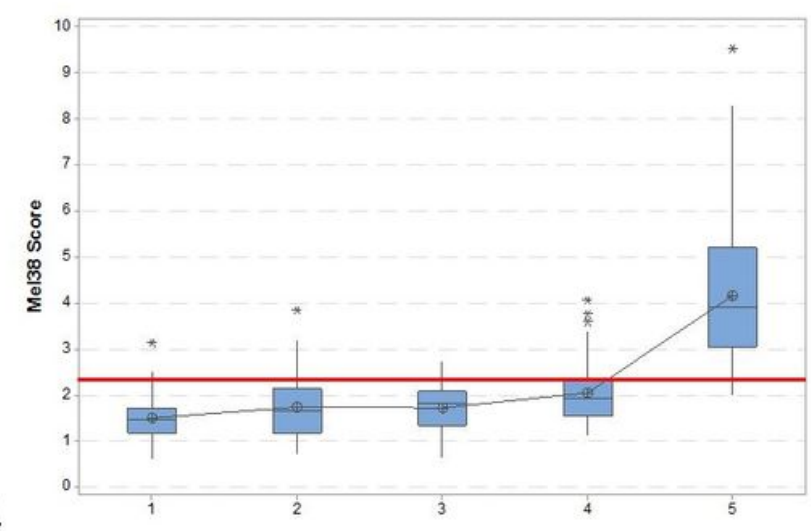

A.

Figure 3 
(A) Box plot of Mel38-FFPE scores grouped by AJCC clinical stage (8th ed). Connecting lines between boxes correspond to mean values. (B) Box plot of Mel38 scores of primary melanocytic lesions grouped by MPATH-Dx melanoma class.

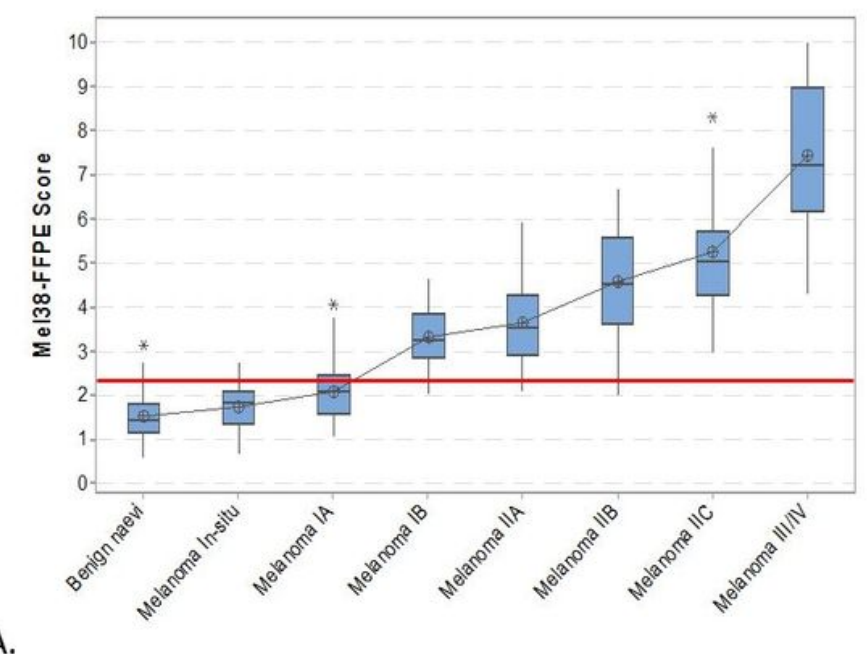

B.

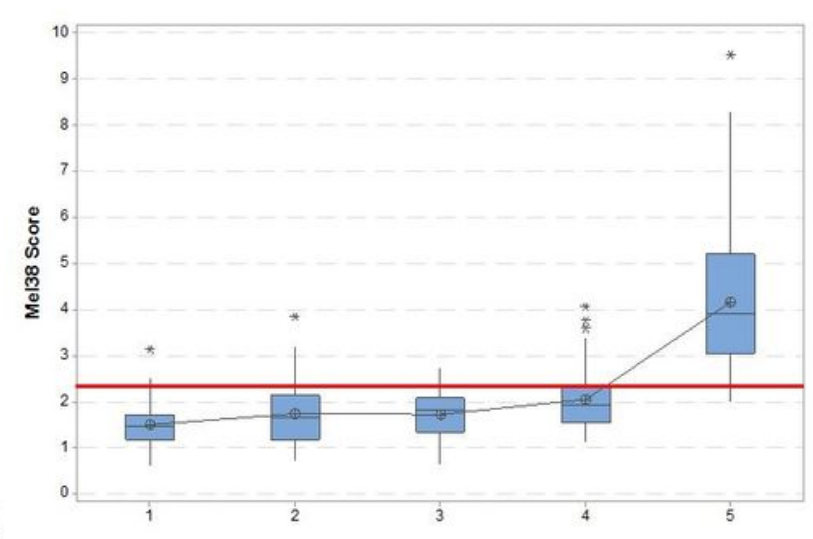

A.

\section{Figure 3}

(A) Box plot of Mel38-FFPE scores grouped by AJCC clinical stage (8th ed). Connecting lines between boxes correspond to mean values. (B) Box plot of Mel38 scores of primary melanocytic lesions grouped by MPATH-Dx melanoma class.

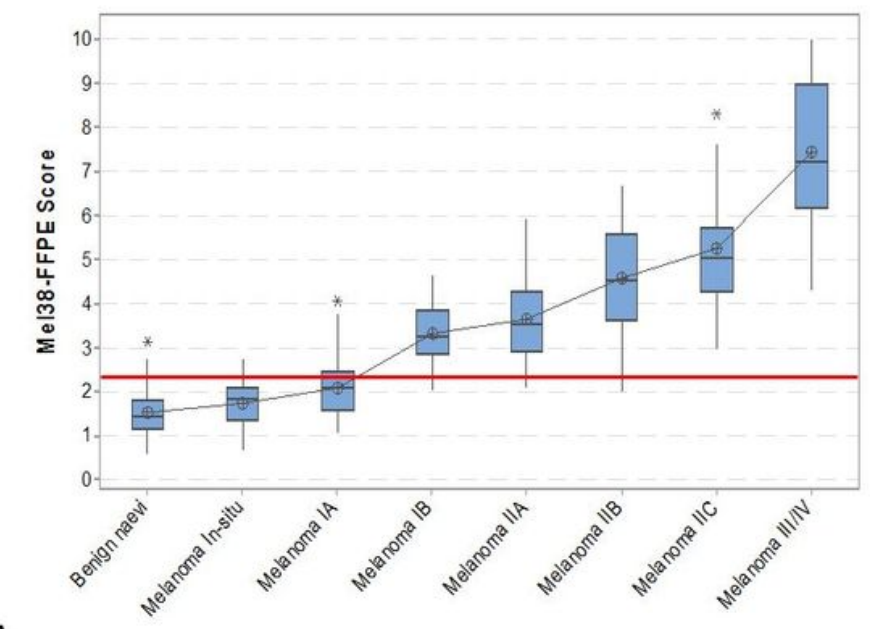

B.

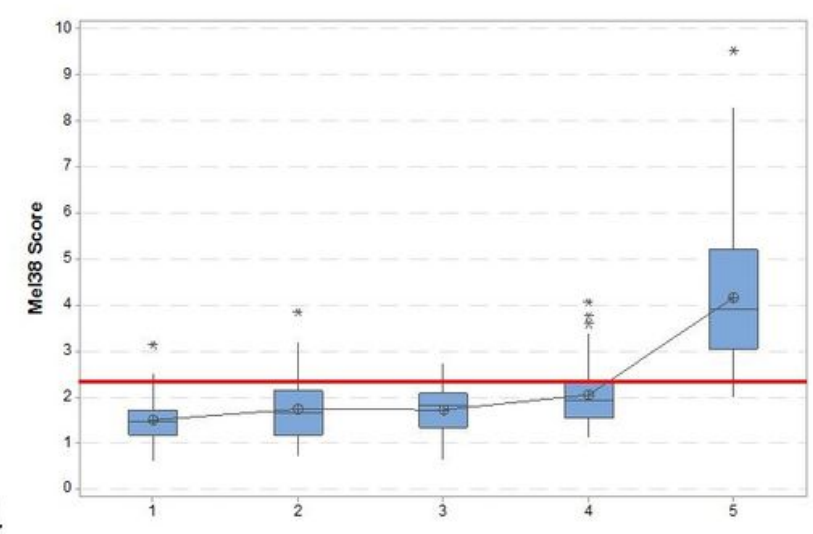

A.

\section{Figure 3}

(A) Box plot of Mel38-FFPE scores grouped by AJCC clinical stage (8th ed). Connecting lines between boxes correspond to mean values. (B) Box plot of Mel38 scores of primary melanocytic lesions grouped by MPATH-Dx melanoma class. 

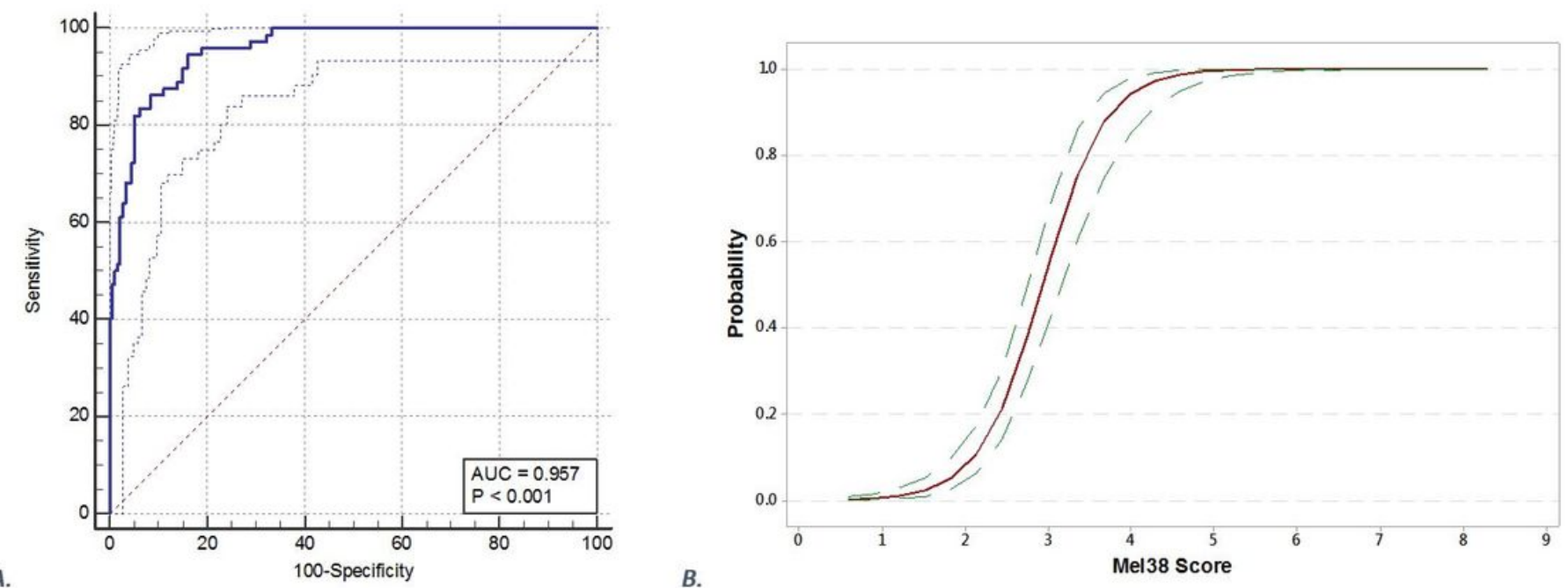

\section{Figure 4}

(A) Receiver operator curve analysis of Mel38 scores from 252 primary melanocytic skin lesions(range 0 to 10) vs. histopathology diagnosis (i.e. MPATH-Dx Classes I- IV vs. Class 5). $95 \% \mathrm{Cl}: 0.93$ to 0.98 . (B) Binary fitted line plot from logistic regression analysis of Mel38 scores indicating the relationship between the genomic score and the corresponding probability of MPATH-Dx Class V melanoma.
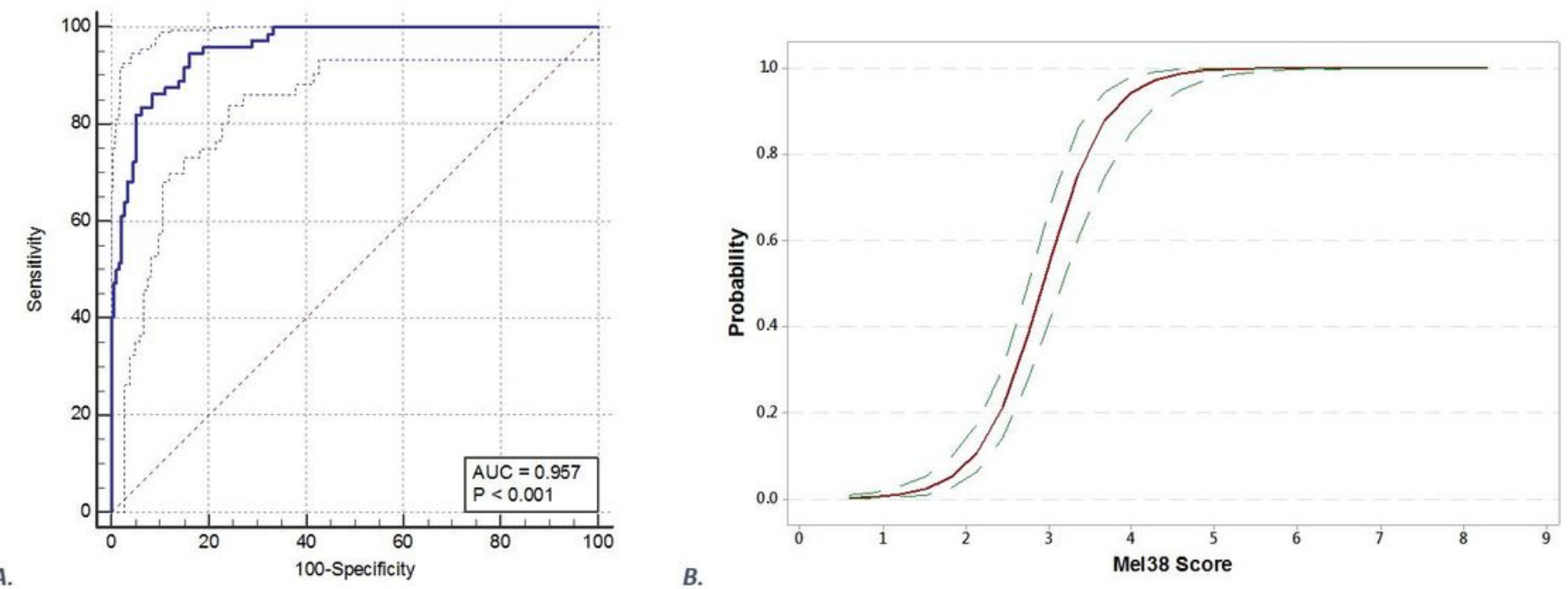

\section{Figure 4}

(A) Receiver operator curve analysis of Mel38 scores from 252 primary melanocytic skin lesions(range 0 to 10 ) vs. histopathology diagnosis (i.e. MPATH-Dx Classes I- IV vs. Class 5). $95 \% \mathrm{Cl}$ : 0.93 to 0.98. (B) Binary fitted line plot from logistic regression analysis of Mel38 scores indicating the relationship between the genomic score and the corresponding probability of MPATH-Dx Class V melanoma. 

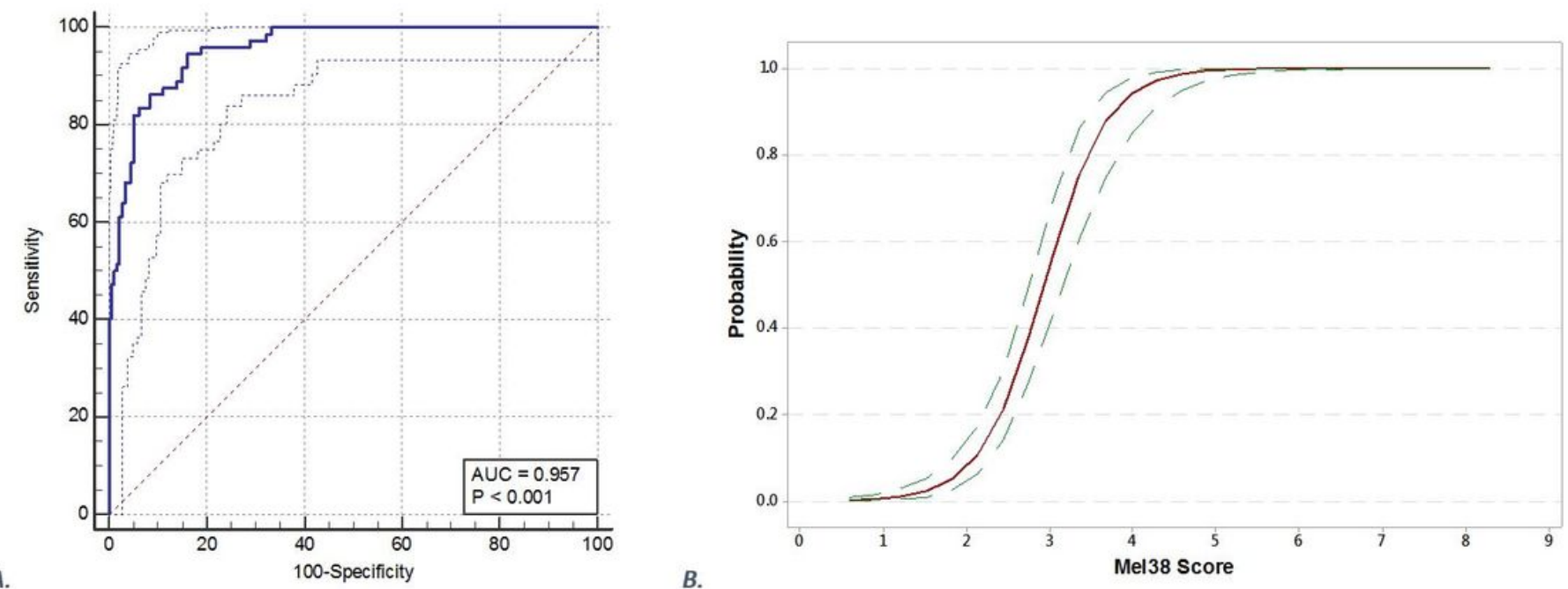

\section{Figure 4}

(A) Receiver operator curve analysis of Mel38 scores from 252 primary melanocytic skin lesions(range 0 to 10) vs. histopathology diagnosis (i.e. MPATH-Dx Classes I- IV vs. Class 5). $95 \% \mathrm{Cl}$ : 0.93 to 0.98. (B) Binary fitted line plot from logistic regression analysis of Mel38 scores indicating the relationship between the genomic score and the corresponding probability of MPATH-Dx Class V melanoma.

A.

Pooled Melanoma RNA control:

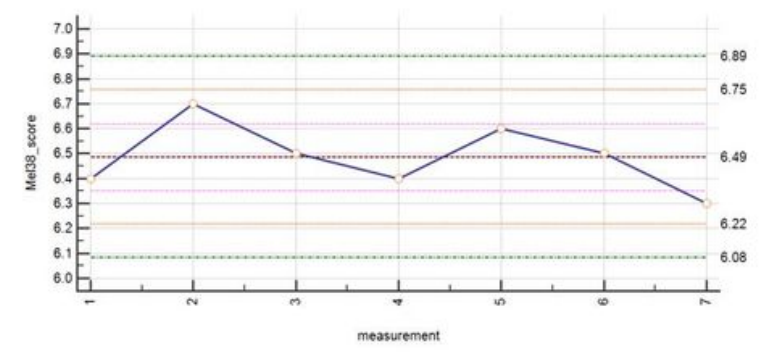

Pooled Naevi RNA control:

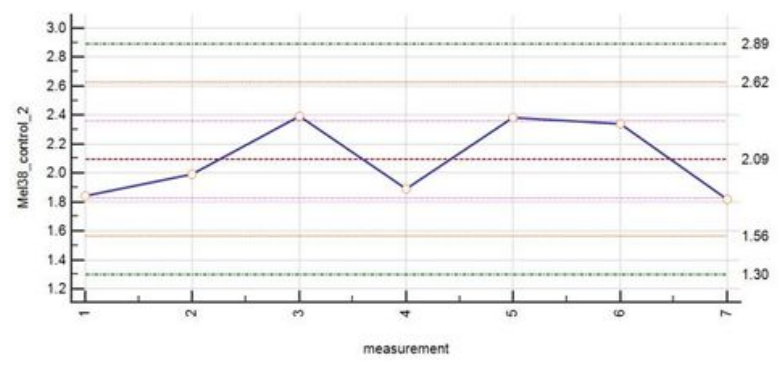

B.

Replicate analysis of individual patient specimens:

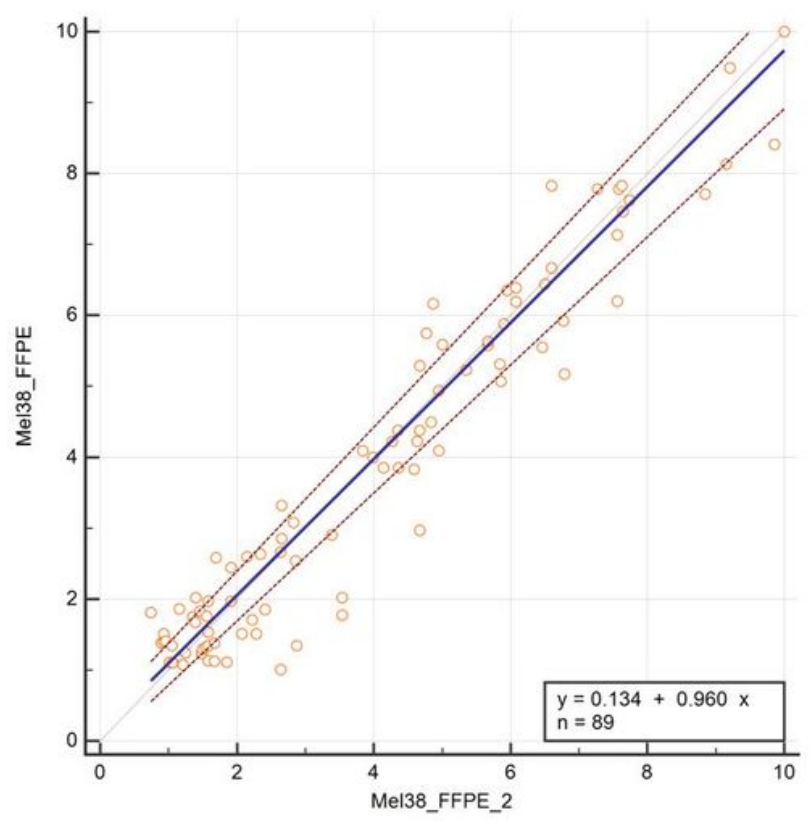

Figure 5

(A) Longitudinal analysis of control sample Mel38 scores. Dashed horizontal lines corresponding to $1 \mathrm{~s}$, 2s and 3s Westguard rules indicated[19]. (B) Passing and Bablok regression of replicate Mel38-FFPE 
specimens. No significant deviation from linearity is detected (Cusom test $P=0.62$ ) and intraclass correlation of 0.96 (0.94 to 0.98 ) reflects the signatures high degree of reproducibility.

A.

Pooled Melanoma RNA control:

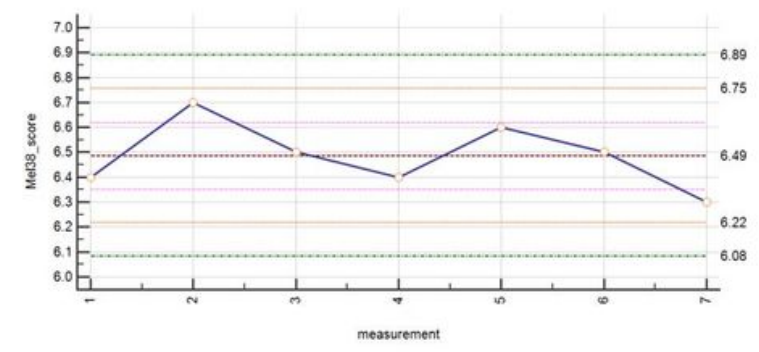

Pooled Naevi RNA control:

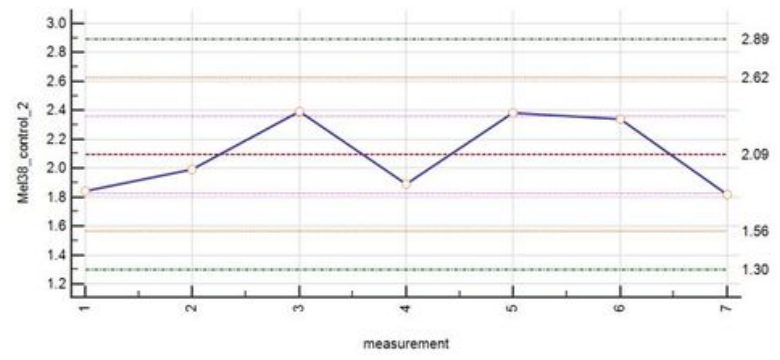

B.

Replicate analysis of individual patient specimens:

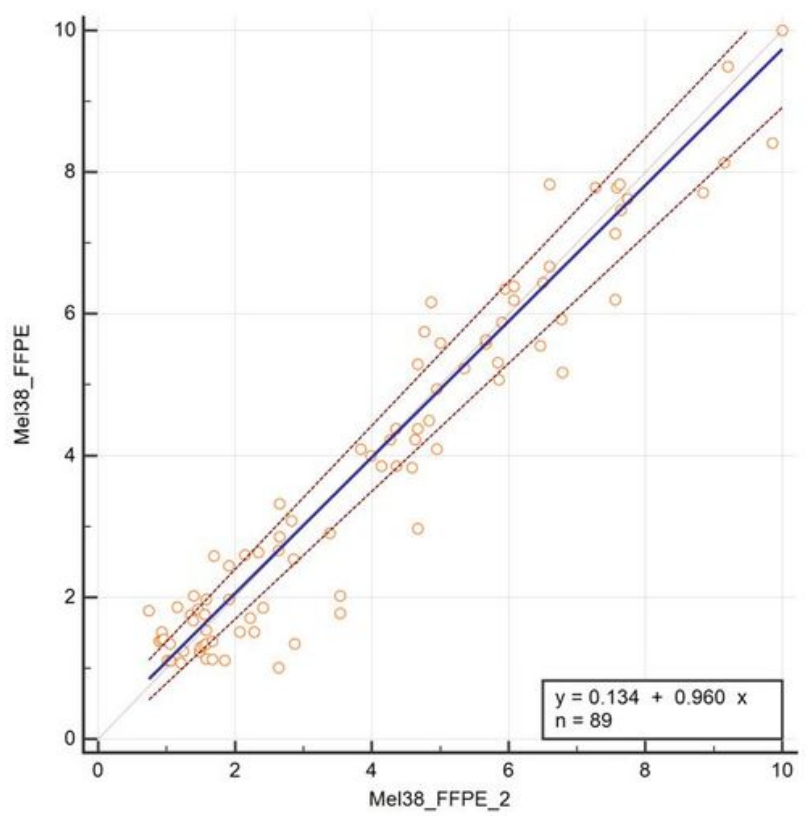

Figure 5

(A) Longitudinal analysis of control sample Mel38 scores. Dashed horizontal lines corresponding to 1s, $2 s$ and $3 s$ Westguard rules indicated[19]. (B) Passing and Bablok regression of replicate Mel38-FFPE specimens. No significant deviation from linearity is detected (Cusom test $\mathrm{P}=0.62$ ) and intraclass correlation of 0.96 (0.94 to 0.98 ) reflects the signatures high degree of reproducibility. 
A.

Pooled Melanoma RNA control:

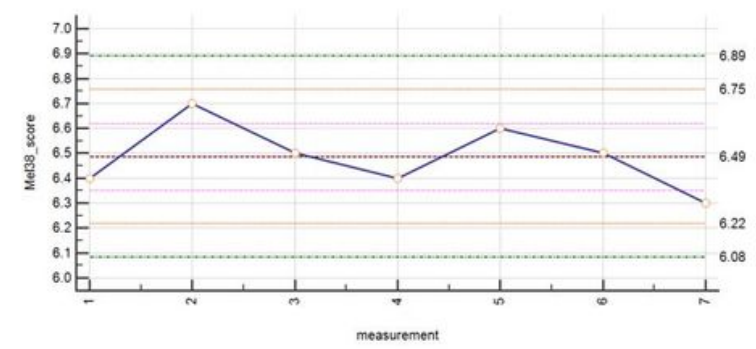

Pooled Naevi RNA control:

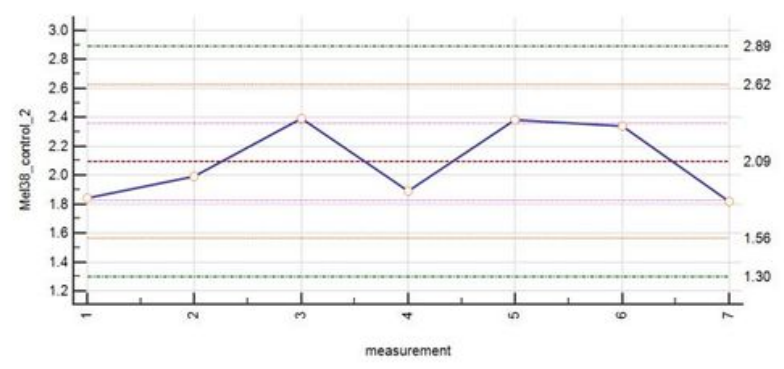

B.

Replicate analysis of individual patient specimens:

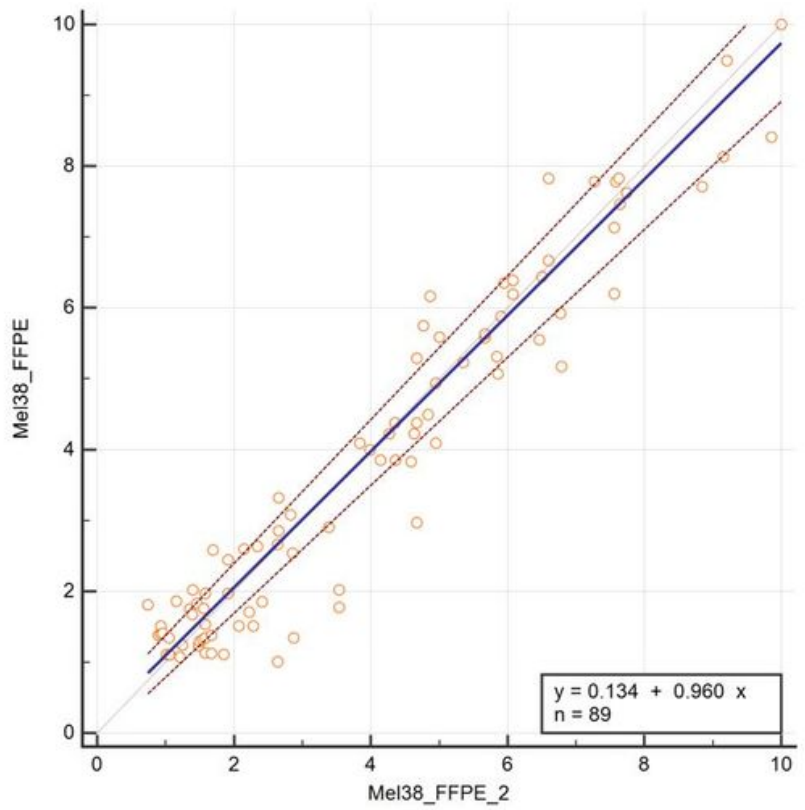

Figure 5

(A) Longitudinal analysis of control sample Mel38 scores. Dashed horizontal lines corresponding to 1s, 2s and 3s Westguard rules indicated[19]. (B) Passing and Bablok regression of replicate Mel38-FFPE specimens. No significant deviation from linearity is detected (Cusom test $\mathrm{P}=0.62$ ) and intraclass correlation of 0.96 (0.94 to 0.98 ) reflects the signatures high degree of reproducibility.

\section{Supplementary Files}

This is a list of supplementary files associated with this preprint. Click to download.

- Supplementarylnformation.docx

- SupplementaryInformation.docx

- Supplementarylnformation.docx 